\title{
PERSISTENT BRADYCARDIA; \\ THE OUTCOME IN PATIENTS WITH BRADYCARDIA AFTER DISCONTINUATION OF RATE SLOWING DRUGS IN TERMS OF FREQUENCY
}

1. FCPS (Card) Assistant Professor Cardiology Faisalabad Institute of Cardiology, Faisalabad, Pakistan.

2. Registrar,

Faisalabad Institute of Cardiology, Faisalabad, Pakistan.

3. PGR FCPS Cardiology, Faisalabad Institute of Cardiology, Faisalabad, Pakistan.

4. Medical officer

Faisalabad Institute of Cardiology, Faisalabad, Pakistan.

Correspondence Address: Dr. Shahid Abbas

Assistant Professor Cardiology

Faisalabad Institute of Cardiology,

Faisalabad, Pakistan.

drshahidabbas@gmail.com

Article received on:

07/05/2017

Accepted for publication:

30/09/2017

Received after proof reading:

$03 / 11 / 2017$

\section{Shahid Abbas', Sehar Fatima², Naeem Hameed ${ }^{3}$, Muhammad Qasim ${ }^{4}$}

ABSTRACT... Objectives: To determine the outcome in patients with bradycardia after discontinuation of rate slowing drugs in terms of frequency of persistent bradycardia. Study Design: Descriptive study. Setting: Faisalabad Institute of Cardiology, Cardiology Department. Period: 2013-2014. Materials and Methods: Pulse and ECG were used to identify the patients and after obtaining informed consent, total 95 patients of bradycardia were included from emergency. Patients with persistent bradycardia were noted after 5 days of discontinuation of culprit drug. Results: Among total 95 patients, 46(48.4\%) were male and 49(51.6\%) were female. Patients with rate limiting drugs include $\beta$ blockers $79(75.8 \%)$, CCBs $19(20 \%)$ and digoxin $4(4.2 \%)$. Patients presented to hospital have sinus bradycardia $3(3.2 \%), 2^{\text {nd }}$ degree AV block $19(20 \%)$ and $3^{\text {rd }}$ degree AV block $73(76.8 \%)$. After 5 days of discontinuation of culprit drug, bradycardia persisted in $32(69.6 \%)$ male, $37(75.5 \%)$ female patients with sinus bradycardia persisted in $1(33.3 \%), 2^{\text {nd }}$ degree AV block in $7(36.8 \%)$ and $3^{\text {rd }}$ degree AV block in $61(83.6 \%)$. Conclusion: In majority of patient on rate slowing drug, bradycardia persisted after discontinuation of these drugs. Bradycardia was not truly drug induced, but it was due to underlying conducting system disease which was unmasked by rate slowing drugs.

Key words: $\quad$ Bradycardia, Persistent Bradycardia, Rate Slowing Drugs, $\beta$-Blocker, Digoxin, Non Dihydropyridine Calcium Channel Blocker, Underlying Conducting System Disease.

Article Citation: Abbas S, Fatima S, Hameed N, Qasim M. Persistent bradycardia; The outcome in patients with bradycardia after discontinuation of rate slowing drugs in terms of frequency. Professional Med J 2017;24(11):1652-1656. DOI:10.17957/TPMJ/17.4229

\section{INTRODUCTION}

The conduction system of heart starts from SA (sinoatrial) node and ends at Purkinjie fibres to cardiac muscles with conduction through Internodal pathways, AV node (Atrio ventricular node), Bundle of HIS, Bundle branches. Sinus node lies at the junction of the right atrium with superior vena cava. ${ }^{1}$ The AV node at the base of inter-atrial septum just above the tricuspid annulus. ${ }^{1}$ The bundle of HIS penetrates the membranous interventricular septum. Bundle of HIS passes through the membranous interventricular septum and divides into Right and Left bundle branch. It divides at the superior margin of muscular interventricular septum, just beneath the membranous septum. In resting adult, SA node rate is $60-100 \mathrm{bpm}$ whereas AV node has intrinsic ability of $40-60$ and ventricles can generate at $30-40 \mathrm{bpm}^{2}$
Bradycardia is present if the heart rate is less than $60 \mathrm{bpm}$ in case of sinus rhythm, atrial rhythms, atrial fibrillation/flutter, junctional, or idioventricular rhythm and atrioventricular (AV) block. ${ }^{3,4}$ Sinus node dysfunction include sinus arrest, sick sinus syndrome, sinus exit block. AV node dysfunction manifest as $1^{\text {st }}$ degree AV block, $2^{\text {nd }}$ degree AV block (Mobitz type $1 \& 2$ ), and $3^{\text {rd }}$ degree AV block or complete heart block. Complete AV block can be due to block at the level of AV node, in the bundle of HIS or below in the purkinji system. ${ }^{5}$ Adults can have phase 3 block (block caused by incomplete action potential recovery) which is rapid rate followed by block. ${ }^{6}$ Hyper-responsiveness of the AV node to vagotonic reflexes results in development of block called paroxysmal AV block. ${ }^{7}$ 
Diagnosis of bradycardia can be made on ECG or holter monitoring. Physical maneuvers like carotid sinus massage and tilt table testing, as well as pharmacologic interventions to test the autonomic reflexes can also be used. Three types of bradycardia may be observed during drug therapy. These include drug-induced bradycardia, drug-provoked bradycardia ${ }^{8,9}$ and drug-associated bradycardia. ${ }^{10}$

Drugs which cause bradycardia include $\beta$ blockers, rate limiting calcium channel blockers and digitalis ${ }^{11}$ with combined drug therapy increases the risk of significant bradycardia. ${ }^{12,13}$ Patients with bradycardia may present with syncope, dizziness, confusion, lightheadedness, fatigue. Patients taking rate limiting drugs and presenting with bradycardia can have underlying conduction system abnormality. In these patients, even after elimination of drug from body, the bradycardia persist. This study was conducted to find out this underlying disease in patients presenting in emergency with symptomatic bradycardia of any type taking rate limiting drugs.

\section{MATERIALS AND METHODS}

This descriptive study was conducted at Faisalabad institute of cardiology, cardiology department in 2013-2014. Pulse and ECG were used to identify the patients and total 95 patients of bradycardia were included from emergency after obtaining informed consent. All enrolled patients were followed everyday by doing ECG and counting pulse twice. Patients in whom bradycardia resolved were discharged.

Patients with persistent bradycardia after 5 days of discontinuation of culprit drug were noted. Patients with age 25-85 years, either sex, on rate slowing drugs like $\beta$-blocker, nondihydropyridine calcium channel blocker, and digoxin and duration of intake of drug one or more than one day were included in study. And patients of Acute MI, digoxin toxicity, bradycardia without rate slowing drugs, patient on chronic amiodarone therapy, hyperklemia and renal failure were excluded. Patient's age, sex, type of block, type of drug and persistence of bradycardia after discontinuation of drug were noted. All these informations collected through prescribed proforma.

\section{STATISTICAL ANALYSIS}

Data was collected on predesigned Proforma by researcher himself. The collected information was analyzed by SPSS software. The confounding variables like age and sex were stratified. Mean and standard deviation calculated for age. Frequency and percentage calculated for sex, type of bradycardia, type of drug used and persistence of bradycardia after 5 days. P-value less than 0.05 taken as significant.

\section{RESULTS}

In the total duration of study of six months, we identified 95 patients with drug induced bradycardia. All patients were taking rate limiting medications i.e. $\beta$-blockers, non-dihydropyridine calcium channel blockers and digoxin which act on AV node.

Mean age was 61 years. Out of 95 patients, $46(48.4 \%)$ were male and $49(51.6 \%)$ were female. Majority of the patients were taking $\beta$-blockers $79(75.8 \%)$, with $19(20 \%)$ on non-dihydropyridine calcium channel blockers and very few were taking digoxin $4(4.2 \%)$. These medications were used for hypertension in many patients.

Most of the patients were admitted with Complete Heart Block 73(76.8\%), with advanced $2^{\text {nd }}$ degree Heart Block in 19(20\%). Majority of the patients had mean heart rate of $30 / \mathrm{min}$ on admission suggesting infra-nodal disease. Sinus bradycardia was in $3(3.2 \%)$.

Rate slowing drugs were discontinued and patients were monitored for 05 days for resolution of bradycardia. On Chi-square analysis there was no gender difference in resolution of bradycardia. However more patient taking $\beta$-blockers had bradycardia that persisted after five days than those taking Calcium Channel Blockers.

Table-I shows the percentage of outcome after 5 days of discontinuation of rate slowing drugs. The persistence of different degrees of AV block after discontinuation of offending drugs was also analyzed. Bradycardia persisted in 32(69.6\%) 
male, $37(75.5 \%)$ female patients with sinus bradycardia in $1(33.3 \%), 2^{\text {nd }}$ degree $\mathrm{AV}$ block in
$7(36.8 \%)$ and $3^{\text {rd }}$ degree AV block in 61(83.6\%).

\begin{tabular}{|l|c|c|c|}
\hline \multicolumn{1}{|c|}{ Characteristics } & $\begin{array}{c}\text { Patients presenting with } \\
\text { bradycardia }\end{array}$ & $\begin{array}{c}\text { Bradycardia persisted after 5 days of } \\
\text { discontinuation of drugs }\end{array}$ & p-value \\
\hline Male & $46(48.4 \%)$ & $32(69.6 \%)$ & 0.516 \\
\hline Female & $49(51.6 \%)$ & $37(75.5 \%)$ & 0.001 \\
\hline Sinus bradycardia & $3(3.2 \%)$ & $1(33.3 \%)$ & 0.001 \\
\hline $2^{\text {nd }}$ degree AV block & $19(20 \%)$ & $7(36.8 \%)$ & 0.001 \\
\hline $3^{\text {rd }}$ degree AV block & $73(76.8 \%)$ & $61(83.6 \%)$ & \\
\hline
\end{tabular}

Table-I. Frequency of persistence of bradycardia after discontinuation of culprit drug

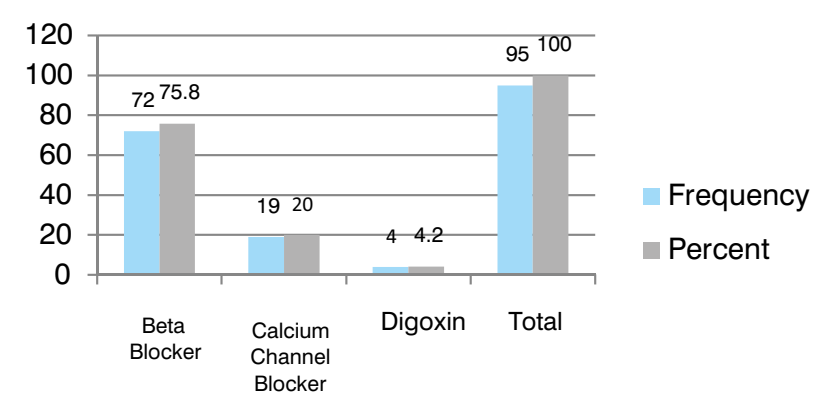

Figure-1. Drugs causing bradycardia with frequency and percentage

\section{DISCUSSION}

Bradycardia associated with rate slowing drugs is a common clinical problem, but the natural history and prognosis of these patients is not well known. We conducted the study at Faisalabad Institute of Cardiology, to clarify the resolution of drug induced bradycardia during hospital stay after discontinuation of rate slowing drugs. Ninety five patients were identified with bradycardia during the study period. Patient with acute MI, digoxin toxicity, bradycardia without rate slowing drugs or due to antiarrhythmic drugs, hyperkalemia and renal failure were excluded. Offending drug was discontinued for five days as it is known that all the drugs are eliminated within their five half lives. Patients were monitored during hospital stay for resolution of bradycardia in the absence of drugs.

After 5 days, bradycardia was not resolved in $72.6 \%$ of patients and resolved in $27.7 \%$ patients. Our data suggested that in majority of the patients the bradycardia persisted even after stopping the offending drug. This is in keeping with other international studies. ${ }^{10}$ This suggested that bradycardia was due to underlying conductive system disease in majority of patients rather than rate slowing drugs. ${ }^{10}$ Older age of our patient also supported it because these patients are more likely to have underlined conduction system disease which was subclinical. ${ }^{14}$

In majority of cases in which bradycardia persisted, there was third degree heart block, which in almost all the cases was of infra-nodal variety. This was expected because all the rate limiting drugs act on AV node or SA node rather than acting on extra-nodal tissues, hence conduction defect in node is more likely to resolve after discontinuation of drugs. This practice would have no effect in case of infra-nodal blocks.

Bradycardia due to drugs was studied on the same population in a recent study..$^{10}$ In patients of $\mathrm{AV}$ block, drugs as sole causative agent was uncommon and observed in only $8 \%$. The majority continued to suffer from AV block even after discontinuation of medications like $\beta$-blocker or calcium channel blockers who presented with $2^{\text {nd }}$ or $3^{\text {rd }}$ degree AV block. Most patients were having infranodal block. It recurred in the absence of drug therapy in many patients after its discontinuation.

On ECG, infra-nodal conduction system disease is diagnosed on QRS width. Underlying conductive system disease is further supported by the persistence of bradycardia in $26.3 \%$ of patients after discontinuation of culprit drug. A QRS width $\geq 120$ ms predicts independently the need of implantation of permanent pace maker in patients of $3^{\text {rd }}$ degree block.

The course of a patient with AV block due to a rate limiting drug would be benign or not, cannot be predicted even after discontinuation of the 
'culprit' medication. Moreover, the 'offending' drug could not be blamed for AV block in many patients, but rather plays a complementary role. In these patients AV block was drug-associated. Only occasionally it was drug-provoked. It is said as drug provoked when the drug plays a trigger role in AV block initiation. The druginduced bradycardia is said to be drug provoked or drug-associated when clinically significant symptomatic bradycardia is either sinus brady or AV nodal bradycardia and caused by any drug.

Thus drug-induced bradycardia is common and frequently it is the subclinical nodal or infranodal conduction system disease by rate slowing drugs which is exposed. True drug-induced bradycardia is caused by drug over dosage and/or drug toxicity or combination of 'drugs-inducing bradycardia' in high doses due to their synergistic effect.

Literature supports that therapeutic doses of drugs, generally, do not cause clinically significant bradycardia in patients with structurally normal hearts. However, in the heart with underlying latent disease of the Sinus and/or AV node, bradycardia may occur due to a triggering effect of the drug. In these cases bradycardia usually develops after prolonged medication use and it may have different underlying mechanisms. The true proarrhythmic events associated with drugs commonly occur within several days from the start of anti-arrhythmic drug therapy at relatively low doses initially. When significant bradycardia is observed in patients taking drugs such as $\beta$-blockers and/or non-DHP calcium channel antagonists, the possibility of significant underlying sinus or conduction system disease or both must be considered.

Clinical difference between drug induced, drug provoked and drug associated bradycardia should be probed deeply but sometimes it is poorly differentiated. Whatever the case is, the need is to avoid bradycardia and symptoms of the patient. Thus in case of clinically significant drug induced bradycardia, one should find out some acceptable alternative to that drug or should reduce the dose of drug. ${ }^{13}$ Pacemaker implantation is an option in patients of bradycardia especially with high degree AV block in case of drug induced, drug provoked or drug associated bradycardia if other measures fail.

Thus bradycardia associated with rate slowing drugs is common clinical problem. In majority of cases the cause is underlying subclinical conduction system disease; which is exposed by rate slowing drugs. In case of bradycardia with extrinsic cause, the removal of offending agent is the first approach to management. ${ }^{15,16}$ Management depends upon symptoms, temporary pacemaker is required in symptomatic bradycardia. If bradycardia is not resolved, PPM may be needed.

\section{LIMITATIONS}

Our diagnosis of infranodal disease depends upon the ECG criteria. We did not study these patients electro physiologically. Due to nature of study, patient's follow up was limited up to their hospital stay. This strategy left a large proportion of patients in whom the bradycardia resolved (27.4\%). Ideally these patients should have been studied by holter monitoring at least two weeks after discharge for resolution or recurrence of bradycardia.

\section{CONCLUSION}

In majority of patient on rate slowing drug i.e. $\beta$-blocker, nondihydopyridine. Calcium channel blockers and digoxin, bradycardia persisted after discontinuation of these drugs. So bradycardia was not truly drug induced, but it was due to underlying conductive system disease which was unmasked by rate slowing drugs.

Copyright (C) 30 Sep, 2017.

\section{REFERENCES}

1. Electrical conduction system of the heart. [Online]. 2010 Oct 21. [cited 2010 Oct21]; URL http://en. wikipedia. org/wiki/Electrical_conduction_system_of_the_Heart.

2. Sanoski CA, Schoen MD, Bauman JL: The arrhythmias. In: Pharmacotherapy: A Pathophysiologic Approach. Seventh Edition. Dipiro JT, Talbert RL, Yee GC, Matzke GR, Wells BG, Posey LM (Eds). New York, The McGrawHill Companies, 2008, pp 279-314.

3. Miller JM, Zipes DP. Management of the patient with cardiac arrhythmias. Braunwald E, Zipes DP, Libby 
P, editors. Heart disease. a textbook of cardiovascular medicine. 6th ed. Philadelphia: WB. Saunders 2001.p.711-39.

4. Lee JH, Ryu HM, Bae MH, Kwon YS, Lee JH, Park Y, et al. Prognosis and natural history of drug-related bradycardia. Korean Circ J. 2009; 39:367-71.

5. Barra SNC, Providência R, Paiva $L$, et al: $A$ review on advanced atrioventricular block in young or middleaged adults. Pacing Clin Electrophysiol 35:1395, 2012.

6. El-Sherif $\mathrm{N}$, Jalife J: Paroxysmal atrioventricular block: Are phase 3 and phase 4 block mecha- nisms or misnomers? Heart Rhythm 6:1514, 2009.

7. Lee S, Wellens HJJ, Josephson ME: Paroxysmal atrioventricular block. Heart Rhythm 6:1229, 2009.

8. Khan MA, Masood N, Husain N, Ahmad B, Aziz T, Naeem $A$, et al. A retrospective study of cardiotoxicities induced by 5-Fluouracil (5-FU) and 5-FU based chemotherapy regimens in Pakistani adult cancer patients at Shaukat Khanum Memorial Cancer Hospital \& Research Center. J Pak Med Assoc. 2012; 62:430-4.

9. Ovsyshcher IE, Barold SS. Drug-induced bradycardia: to pace or not to pace? Pacing Clin Electrophysiol. 2004; 27:1144-47.
10. Zeltser D, Justo D, Halkin A, Rosso R, ISh-shalom M, Hochenberg $M$, et al. Drug-induced atrioventricular block: prognosis after discontinuation of the culprit drug. J Am Coll Cardiol.2004; 44:105-8.

11. Ovsyshcher IE, Barold SS (2004) Drug-induced bradycardia: to pace or not to pace? Pacing Clin Electrophysiol 27:1144-1147.

12. Yusuf S, Camm AJ (2003) Sinus tachyarrhythmias and the specific bradycardia agents: a marriage made in heaven? J Cardiovasc Pharmacol Ther 8:89-105.

13. Hauser TH, Pinto DS, Josephson ME et al (2003) Safety and feasibility of a clinical pathway for the outpatient initiation of antiarrhythmic medications in patients with atrial fibrillation or atrial flutter. Am $\mathrm{J}$ Cardiol 91:1437-1441.

14. Lee JH, Ryu HM, Bae MH, Kwon YS, Lee JH, Park Y, et al. Prognosis and natural history of drug-related bradycardia. Korean Circ J. 2009; 39:367-71.

15. Gupta AK, Maheshwari A, Tresch DD, Thakur RK. Cardiac arrhythmias in the elderly. Card Electrophysiol Rev 2002; 6:120-128.

16. Shohat-Zabarski R, lakobishvili Z, Kusniec J, Mazur A, Strasberg B. Paroxysmal atrioventricular block: clinical experience with 20 patients. Int $\mathrm{J}$ Cardiol 2004; 97:399-405.

\begin{tabular}{|c|c|c|c|}
\hline \multicolumn{4}{|c|}{ AUTHORSHIP AND CONTRIBUTION DECLARATION } \\
\hline Sr. \# & Author-s Full Name & Contribution to the paper & Author $=\mathbf{s}$ Signature \\
\hline 1 & Shahid Abbas & 1st Author & \\
\hline 2 & Sehar Fatima & 2nd Author & \\
\hline 3 & Naeem Hameed & 3rd Author & \\
\hline 4 & Muhammad Qasim & 4th Author & \\
\hline
\end{tabular}

